\title{
ON THE GAPS BETWEEN NON-ZERO FOURIER COEFFICIENTS OF EIGENFORMS WITH CM
}

\author{
SURJEET KAUSHIK AND NARASIMHA KUMAR
}

\begin{abstract}
Suppose $E$ is an elliptic curve over $\mathbb{Q}$ of conductor $N$ with complex multiplication $(\mathrm{CM})$ by $\mathbb{Q}(i)$, and $f_{E}$ is the corresponding cuspidal Hecke eigenform in $S_{2}^{\text {new }}\left(\Gamma_{0}(N)\right)$. Then $n$-th Fourier coefficient of $f_{E}$ is non-zero in the short interval $\left(X, X+c X^{\frac{1}{4}}\right)$ for all $X \gg 0$ and for some $c>0$. As a consequence, we produce infinitely many cuspidal CM eigenforms $f$ level $N>1$ and weight $k>2$ for which $i_{f}(n) \ll n^{\frac{1}{4}}$ holds, for all $n \gg 0$.
\end{abstract}

\section{INTRODUCTION}

Estimating the size of possible gaps between the non-zero Fourier coefficients of modular cuspforms is one of the fundamental and interesting object of study in number theory. In this short note, we are interested in a question of Serre in bounding the maximum length of consecutive zeros of elliptic curves with CM by $\mathbb{Q}(i)$ and cusp forms of higher weight and level.

A famous conjecture of Lehmer predicts that $\tau(n) \neq 0$ for any $n \geq 1$, where

$$
\Delta(z)=\sum_{n=1}^{\infty} \tau(n) q^{n}
$$

is the unique normalized cuspidal eigenform of weight 12 and level 1. In a relation to Lehmer's conjecture, in [Ser81, Serre initiated the general study of estimating the size of possible gaps between the non-zero Fourier coefficients of modular cuspforms by the function

$$
i_{f}(n):=\max \left\{i: a_{f}(n+j)=0 \text { for all } 0 \leq j \leq i\right\} .
$$

In fact, he proved that if $f(z)$ is a cusp form of weight $k \geq 2$ which is not a linear combination of forms with $\mathrm{CM}$, then

$$
i_{f}(n) \ll n .
$$

In that article, he also poses a question, if the bound can be improved to $n^{\delta}$ with $0<\delta<1$. In [BO01, Balog and Ono proved that, for a cuspidal eigenform on $\Gamma_{0}(N)$, not a linear combination of forms with $\mathrm{CM}$, that for any $\epsilon>0$,

$$
i_{f}(n) \ll_{f, \epsilon} n^{17 / 41+\epsilon} \quad \forall n \in \mathbb{N} .
$$

Currently, the best bound for $i_{f}(n)$ is available due to Kowalski, Robert, and Wu and they proved that for any holomorphic non-CM cuspidal eigenform $f$ on general congruence groups,

$$
i_{f}(n) \ll_{f} n^{7 / 17+\epsilon}
$$

2010 Mathematics Subject Classification. Primary 11F30; Secondary 11F11, 11F33, 11G05.

Key words and phrases. Elliptic curves, Fourier coefficients of cuspidal eigenforms, CM eigenforms, modular forms of higher weight. 
holds for all $n \in \mathbb{N}$ (cf. [KRW07]). However, in the case of elliptic curves, the best bound is available due to Alkan. In [Alk03], he showed that, if $f$ is a weight 2 cusp form corresponding to an elliptic curve without $\mathrm{CM}$, then

$$
i_{f}(n) \ll_{f, \epsilon} n^{\frac{69}{169}+\epsilon} .
$$

Through various approaches, many mathematicians have contributed in answering this question. The approaches are either using Rankin-Selberg estimates, or Chebotarev density theorem, or distribution of $B$-free numbers, etc., (cf.[BO01], [Alk03, Alk05], [AZ05a], AZ05b], [Mat12], KRW07], AZ08]).

In [AZ05c], for the first time, the authors have exploited the idea of using congruences to study $i_{f}(n)$. There it was shown that $i_{\Delta}(n) \ll n^{\frac{1}{4}}$, where $\Delta$ is the unique normalized cuspidal eigenform of weight 12. The proof of this theorem relies on the existance of sums of squares in short intervals of the form $\left(x, x+x^{\frac{1}{4}}\right)$. In [DG14, the authors extended the above idea to show that for any non-zero modular form $f \in S_{k}\left(\Gamma_{0}(1)\right)$ with $k \geq 12$, one has

$$
i_{f}(n) \ll n^{1 / 4}
$$

$\forall n \gg 0$, where the implied constant depends only on $k$.

If the level $N>1$, then there are no similar general results are available with $i_{f}(n) \ll n^{\frac{1}{4}}$. However, in DG15, the authors were able to produce infinitely many non-isogenous elliptic curves for which (1.2) holds. In [Kum16], the second author had constructed examples of non-CM (and CM) cuspidal eigenform of level $N>1$ and weight $k>2$ for which the (1.2) holds.

In the approaches through Chebotarev density theorem, or distribution of $B$-free numbers, etc.., one need to assume that the cuspidal eigenforms are without CM. This is because, all those approaches depends on a result of Serre, which is valid only for eigenforms without CM. However, the analogous question make sense for cuspidal eigenforms with CM as well, and this can be handled through the approach of [AZ05c], DG14, DG15], Kum16].

In this short note, we show that any elliptic curve over $\mathbb{Q}$ with $\mathrm{CM}$ by $\mathbb{Q}(i)$ satisfy

$$
i_{f}(n) \ll n^{\frac{1}{4}}
$$

for $n \gg 0$. Then, we shall produce a one-parameter family of elliptic curves over $\mathbb{Q}$ with CM by $\mathbb{Q}(i)$ and infinitely many cuspidal CM eigenforms level $N>1$ and weight $k>2$ for which the (1.2) holds.

\section{Statements AND PROOFS}

Suppose $E$ is an elliptic curve over $\mathbb{Q}$ of conductor $N$ with CM by $\mathbb{Q}(i)$. Let $f_{E} \in S_{2}^{\text {new }}\left(\Gamma_{0}(N)\right)$ be the corresponding cuspidal eigenform of level $N$, by the modularity theorem. In this section, we shall show that the elliptic curve $E$ satisfies

$$
i_{f_{E}}(n) \ll n^{\frac{1}{4}}
$$

for $n \gg 0$.

Let us briefly recall the notion of CM. Let $K$ be an imaginary quadratic field and $\mathcal{O}_{K}$ be the integral closure. Let $\mathfrak{m}$ be an integral ideal of $K$ and let $I(\mathfrak{m})$ be the group of fractional ideals of $K$ co-prime to $\mathfrak{m}$. By definition, a Hecke character $\Psi$ of $K$ is a homomorphism

$$
\Psi: I(\mathfrak{m}) \rightarrow \mathbb{C}^{*}
$$


such that $\Psi(\alpha)=\alpha^{r}$ for all $\alpha \in K^{*}$ with $\alpha \equiv 1 \bmod \mathfrak{m}$. For such a Hecke character $\Psi$, one can associate the function $f=f_{\psi}$ defined by

$$
f_{\psi}(z)=\sum_{\mathfrak{a} \subseteq \mathcal{O}_{K},(\mathfrak{a}, \mathfrak{m})=1} \Psi(\mathfrak{a}) e^{2 \pi i(N \mathfrak{a}) z},
$$

where $N \mathfrak{a}$ denotes the norm of $\mathfrak{a}$. We can also write the function as

$$
f_{\psi}(z)=\sum_{n \geq 1} a_{\psi}(n) e^{2 \pi i n z}
$$

where

$$
a_{\psi}(n)=\sum_{(\mathfrak{a}, \mathfrak{m})=1, N \mathfrak{a}=n} \Psi(\mathfrak{a})
$$

By a theorem of Hecke, $f_{\psi} \in S_{r+1}\left(\Gamma_{0}\left(\left|d_{K}\right| N \mathfrak{m}\right), \epsilon\right)$ is an eigenform, where $d_{K}$ is discriminant of field $K, \epsilon$ is a character modulo $\left|d_{K}\right| N \mathfrak{m}$ (cf. refer to Rib77] for more details). Let us recall some useful results from [DG14, Theorem 1] and [KRW07, Lemma 2.2], which we shall use.

Theorem 2.1 (Das-Ganguly). Given any integer $N \in \mathbb{N}$, there exists $X_{0} \in \mathbb{R}^{+}$and $c>0$ (depending only on $N$ ) such that there exists an integer $n$ which is a sum of two squares and co-prime to $N$ in intervals of type $\left(X, X+c X^{\frac{1}{4}}\right)$ for all $X \gg X_{0}$.

Lemma 2.2 (Kowalski-Robert-Wu). If $f=\sum_{n=1}^{\infty} a_{f}(n) q^{n}$ is a normalized cuspidal eigenform in $S_{2 k}\left(\Gamma_{0}(N), \chi\right)$, then there exists a natural number $M_{f} \geq 1$ such that for any prime $p \nmid M_{f}$, either $a_{f}(p)=0$ or $a_{f}\left(p^{r}\right) \neq 0$ for all $r \geq 1$. If $\chi$ is trivial and $f$ has integer coefficients, then one can take $M_{f}=N$.

Now, let us recall Deuring's theorem for elliptic curves over $\mathbb{Q}$ (cf. Lan73, Chapter 13, Theorem 12]).

Theorem 2.3 (Deuring). Let $E$ be an elliptic curve over $\mathbb{Q}$ with $C M$ by $K$. Let $\Psi$ be the corresponding Hecke character. If $p \geq 5$, then the number $a_{E}(p)$ is zero if and only if either $p$ is inert or ramified in $K$ or divides the conductor of $\Psi$ (equivalently, the elliptic curve has bad reduction).

Now, we are ready to prove the theorem that we are alluded to in the beginning of this section.

Theorem 2.4. Let $E$ be an elliptic curve over $\mathbb{Q}$ of conductor $N$ with $C M$ by $\mathbb{Q}(i)$ and $f \in S_{2}^{\text {new }}\left(\Gamma_{0}(N)\right)$ be the corresponding cuspidal eigenform. Then,

$$
i_{f}(n) \ll n^{\frac{1}{4}}
$$

for $n \gg 0$, where the implied constant depends on $E$.

Proof. Since $E$ is an elliptic curve over $\mathbb{Q}$ with $C M$, let $\Psi$ be the corresponding Hecke character. Take $N=6 M_{f} \operatorname{cond}(\Psi)$, where $M_{f}$ as in Lemma 2.2. By Theorem 2.1, there exists $X_{0} \in \mathbb{R}$ and $c>0$ (depending only on $N$ ) such that there exists an integer, say $m$, which is a sum of two squares and co-prime to $N$ in intervals of type $\left(X, X+c X^{\frac{1}{4}}\right)$ for all $X \gg X_{0}$. So, to prove the theorem, it is sufficient to show that $a_{f}(m)$ is non-zero.

Since $m$ is a sum of squares and $(m, 6)=1, m$ can be written as

$$
m=p_{i} \equiv 1 \prod_{(\bmod 4)} p_{i}^{r_{i}} \prod_{q_{i} \equiv 3} \prod_{(\bmod 4)} q_{i}^{2 s_{i}} .
$$


By (2.2), we see that for inert primes $q$ of $\mathbb{Q}(i)$, the Fourier coefficients $a_{f}(q)$ are zero because there are no ideals of norm $q$. By quadratic reciprocity law, the odd primes $q$ which remain inert in $\mathbb{Q}(i)$ are exactly the primes $q \equiv 3(\bmod 4)$. However, the power of $q_{i}$ in $m$ are even and we show that $a_{f}\left(q_{i}^{2 s_{i}}\right)$ is non-zero. This is because, the Hecke relations would imply that

$$
a_{f}\left(q_{i}^{r}\right)=a_{f}\left(q_{i}\right) a_{f}\left(q_{i}^{r-1}\right)-q_{i} a_{f}\left(q_{i}^{r-2}\right)
$$

would imply that $a_{f}\left(q_{i}^{2 r}\right)=\left(-q_{i}\right)^{r} a_{f}\left(q_{i}^{2 r-2}\right)$. Since $a_{f}\left(q_{i}^{2}\right)$ is non-zero, we see that $a_{f}\left(q_{i}^{2 r}\right)$ 's are also non-zero, for all $r \geq 1, i \geq 1$.

For any split prime $p$ of $\mathbb{Q}(i)$, the Fourier coefficient $a_{f}(p)$ is non-zero, by Theorem 2.3 and $(m, 6$ cond $(\Psi))=1$. This implies that $a_{f}\left(p^{r}\right) \neq 0$ for all $r \geq 1$, since $\left(m, M_{f}\right)=1$ and by Lemma 2.2. This shows that, for $p \equiv 1(\bmod 4)$, we have $a_{f}\left(p^{r}\right) \neq 0$ for all $r \geq 1$, since the odd primes $p \equiv 1(\bmod 4)$ are exactly the split primes of $\mathbb{Q}(i)$.

Hence

$$
a_{f}(m)=\prod_{p_{i} \equiv 1} \prod_{(\bmod 4)} a_{f}\left(p_{i}^{r_{i}}\right) \prod_{q_{i} \equiv 3} \prod_{(\bmod 4)} a_{f}\left(q_{i}^{2 s_{i}}\right) \neq 0,
$$

hence we are done with the proof.

Remark 2.5. The crux in the proof of Theorem 2.4 is Deuring's Theorem. We could have a theorem similar to that of Theorem 2.4 for eigenforms of weight $2 k$ with $k>1$, if we had an analogous result of Deuring for higher weights. But, the authors are not aware of such results.

Remark 2.6. One might wonder the reason for working with $\mathbb{Q}(i)$, but not with any other imaginary quadratic fields. The split (resp. inert) primes of $\mathbb{Q}(i)$ and the primes $p \equiv 1(\bmod 4)(\operatorname{resp} ., p \equiv 3(\bmod 4))$ occur in the decomposition of sums of squares have a relation. In fact, the primes $p \equiv 1(\bmod 4)$ are exactly the split primes. This fact, we have used it in the proof of the Theorem 2.4.

There exists infinitely many elliptic curves $E / \mathbb{Q}$ with $\mathrm{CM}$ by $\mathbb{Q}(i)$. For example, one could take a one parameter family of elliptic curves $y^{2}=x^{3}+a x$, with $a$ varies over $\mathbb{Q}^{*}$. In this case, the endomorphism ring of $E$ is $\mathbb{Z}[i]$. However, these curves are isogenous, since any two CM elliptic curves with the same endomorphism algebra are isogenous.

We shall finish this note with the following proposition.

Proposition 2.7. There exists infinitely many eigenforms with $C M$ of weight $k>2$ and level $N>1$ for which (1.2) holds.

Proof. Let $E$ be an elliptic curve over $\mathbb{Q}$ with $\mathrm{CM}$ by $\mathbb{Q}(i)$. Let $\Psi$ be the corresponding Hecke character. Consider the Hecke character $\Psi^{m}$, for some odd $m$. By a result of Hecke, the corresponding $f_{\Psi^{m}}$ is an eigenform with $\mathrm{CM}$ of weight $m+1$ with trivial character. However, the eigenform $f_{\Psi^{m}}$ may not be a newform unless $\Psi^{m}$ is primitive.

By [Rib77, Cor. 3.5], corresponding to $f_{\Psi^{m}}$, there exists a unique newform with CM, which we denote with $g_{m}=\sum_{n=1}^{\infty} a_{g_{m}}(n) q^{n}$. Then, the newform $g_{m}$ of weight $m+1$ with trivial character and level dividing the level of $f_{\Psi^{m}}$. Moreover, $g_{m}(z)$ has the property that $a_{g_{m}}(p)=a_{\Psi^{m}}(p)$ for primes $p$ away from the level of $f_{\Psi^{m}}$. Therefore, $a_{g_{m}}(p)=0$ if and only if $a_{\Psi^{m}}(p)=0$, for all but finitely many primes. By [LK14, Prop. 5.1], if $p \geq 5$, we see that $a_{\Psi^{m}}(p)=0$ if and only if $a_{\Psi}(p)=0$. 
By arguing as in the proof of Theorem 2.4, we see that $i_{g_{m}}(n) \ll n^{\frac{1}{4}}$. Hence, there exists infinitely many eigenforms with CM of different weights for which (1.2) holds. The levels of $g_{m}$ 's are $>1$ because eigenforms inside $S_{k}\left(\mathrm{SL}_{2}(\mathbb{Z})\right)$ are without CM, for any weight $k$.

\section{REFERENCES}

[Alk03] Alkan, Emre. Nonvanishing of Fourier coefficients of modular forms. Proc. Amer. Math. Soc. 131 (2003), no. 6, 1673-1680

[Alk05] Alkan, Emre. On the sizes of gaps in the Fourier expansion of modular forms. Canad. J. Math. 57 (2005), no. 3, 449-470.

[AZ05a] Alkan, Emre; Zaharescu, Alexandru. Nonvanishing of Fourier coefficients of newforms in progressions. Acta Arith. 116 (2005), no. 1, 81-98.

[AZ05b] Alkan, Emre; Zaharescu, Alexandru. $B$-free numbers in short arithmetic progressions. J. Number Theory 113 (2005), no. 2, 226-243.

[AZ05c] Alkan, Emre; Zaharescu, Alexandru. Nonvanishing of the Ramanujan tau function in short intervals. Int. J. Number Theory 1 (2005), no. 1, 45-51.

[AZ07] Alkan, Emre. Average size of gaps in the Fourier expansion of modular forms. Int. J. Number Theory 3 (2007), no. 2, 207-215.

[AZ08] Alkan, Emre; Zaharescu, Alexandru. On the gaps in the Fourier expansion of cusp forms. Ramanujan J. 16 (2008), no. 1, 41-52.

[BO01] Balog, Antal; Ono, Ken. The Chebotarev density theorem in short intervals and some questions of Serre. J. Number Theory 91 (2001), no. 2, 356-371.

[DG14] Das, Soumya; Ganguly, Satadal. Gaps between nonzero Fourier coefficients of cusp forms. Proc. Amer. Math. Soc. 142 (2014), no. 11, 3747-3755.

[DG15] Das, Soumya; Ganguly, Satadal. A note on small gaps between nonzero Fourier coefficients of cusp forms. Proc. Amer. Math. Soc. 144 (2016), no. 6, 2301-2305.

[DS05] Diamond, Fred; Shurman, Jerry. A first course in modular forms. Graduate Texts in Mathematics, 228. Springer-Verlag, New York, 2005.

[KRW07] Kowalski, Emmanuel; Robert, Olivier; Wu, Jie. Small gaps in coefficients of $L$-functions and $B$-free numbers in short intervals. Rev. Mat. Iberoam. 23 (2007), no. 1, 281-326.

[Kum16] Kumar, Narasimha. On the gaps between non-zero Fourier coefficients of cusp forms of higher weight. To appear in the Ramanujan Journal.

[Lan73] Lang, Serge. Elliptic functions. Addison-Wesley Publishing Co., Inc., Reading, Mass.London-Amsterdam, 1973.

[LK14] Laptyeva, N.; Kumar Murty, V. Fourier coefficients of forms of CM-type. Indian J. Pure Appl. Math. 45 (2014), no. 5, 747-758.

[Mat12] Matomäki, Kaisa. On the distribution of $B$-free numbers and non-vanishing Fourier coefficients of cusp forms. Glasg. Math. J. 54 (2012), no. 2, 381-397.

[Ras09] Rasmussen, Jonas B. Higher congruences between modular forms. PhD Thesis, Department of Mathematical Sciences, University of Copenhagen - 2009.

[Rib77] Kenneth A. Ribet. Galois representations attached to eigenforms with Nebentypus. In Modular functions of one variable, V (Proc. Second Internat. Conf., Univ. Bonn, Bonn, 1976), pages 1751. Lecture Notes in Math., Vol. 601. Springer, Berlin, 1977.

[Ser81] Serre, Jean-Pierre. Quelques applications du théorème de densité de Chebotarev. Inst. Hautes tudes Sci. Publ. Math. No. 54 (1981), 323-401.

E-mail address: ma15resch01001@iith.ac.in

Department of Mathematics, Indian Institute of Technology Hyderabad, Kandi, SANGAREDDY - 502285, INDIA.

E-mail address: narasimha.kumar@iith.ac.in

Department of Mathematics, Indian Institute of Technology Hyderabad, Kandi, SANGAREDDY - 502285, INDIA. 